\title{
Modeling Droplet Heat and Mass Transfer during Spray Bar Pressure Control of the Multipurpose Hydrogen Test Bed (MHTB) Tank in Normal Gravity
}

\author{
O. Kartuzova and M. Kassemi \\ National Center for Space Exploration Research (NCSER) \\ NASA Glenn Research Center \\ Cleveland, $\mathrm{OH} 44135$
}

\begin{abstract}
A CFD model for simulating pressure control in cryogenic storage tanks through the injection of a subcooled liquid into the ullage is presented and applied to the $1 \mathrm{~g}$ MHTB spray bar cooling experiments. An Eulerian-Lagrangian approach is utilized to track the spray droplets and capture the interaction between the discrete droplets and the continuous ullage phase. The spray model is coupled with the VOF model by performing particle tracking in the ullage, removing particles from the ullage when they reach the interface, and then adding their contributions to the liquid. A new model for calculating the droplet-ullage heat and mass transfer is developed. In this model, a droplet is allowed to warm up to the saturation temperature corresponding to the ullage vapor pressure, after which it evaporates while remaining at the saturation temperature. The droplet model is validated against the results of the MHTB spray-bar cooling experiments with $50 \%$ and $90 \%$ tank fill ratios. The predictions of the present T-sat based model are compared with those of a previously developed kinetic-based droplet mass transfer model. The predictions of the two models regarding the evolving tank pressure and temperature distributions, as well as the droplets' trajectories and temperatures, are examined and compared in detail. Finally, the ullage pressure and local vapor and liquid temperature evolutions are validated against the corresponding data provided by the MHTB spray bar mixing experiment.
\end{abstract}
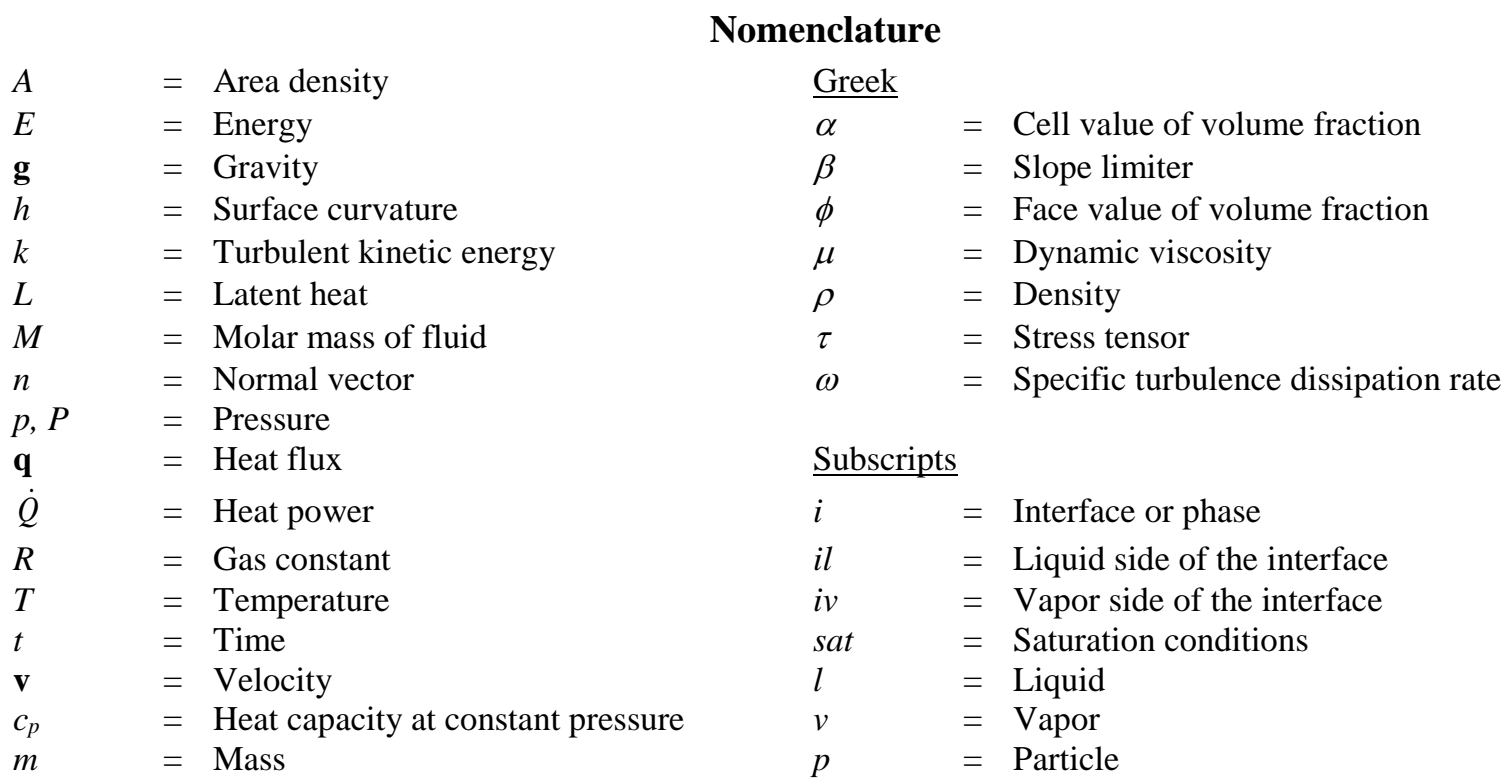

\section{Introduction}

Efficient cryogenic fluid management of high energy propellants ${ }^{1}$ is a crucial enabling technology in the path of nearly all future NASA human exploration mission scenarios. The main challenge for the long duration 
storage of cryogens in space is self-pressurization caused by heat leaks through the insulation and along various conduction paths. Thrusters have traditionally been used to settle the propellant and relieve the tank pressure by venting during short term operations. For long duration missions, the added propellant and hardware weight to accommodate the increasing number of venting cycles will be quite prohibitive. Thus, maintaining the tank pressure while minimizing the boil-off loss of propellants through an active pressure control mechanism has become a significant challenge associated with the long term storage of cryogens in microgravity ${ }^{2}$.

Among various pressure control strategies that have been proposed and tested, the spray-bar thermodynamic vent system (TVS) has emerged as a promising mechanism that enables tank pressure control through reduced venting without resettling. The key components of a TVS include a Joule-Thomson (J-T) expansion, a two-phase heat exchanger, and a spray-bar mixing pump system. These components enable thermal destratification and the extraction of heat from the tank with minimized liquid cryogen losses ${ }^{3}$ during pressure control. For future microgravity applications, a demonstration of a spray-bar TVS under unsettled conditions is needed, and the optimization of this pressure control system for successful performance under weightlessness conditions is required. In this regard, Computational Fluid Dynamics (CFD) models that have been validated against ground-based test data can serve as useful design tools that can extrapolate and optimize the ground-tested spray-bar TVS design for successful microgravity operations. Development and validation of such analysis tools also falls within the main objectives of the NASA Evolvable Cryogenics project to provide a computational design platform for cryogenic fluid management techniques and equipment under settled and unsettled conditions.

Numerous numerical models with different degrees of sophistication have been developed to study storage tank operations in $1 \mathrm{~g}$ and/or microgravity ${ }^{4}$. However, many of these models have only studied tank selfpressurization $^{5-7}$. A few have also investigated tank pressure control, but mainly for applications where an axial forced jet is used to perform thermal destratification through liquid mixing ${ }^{8-11}$. In the Multipurpose Hydrogen Test Bed experiment, storage tank pressure control is accomplished through the action of the longitudinal spray-bar Thermodynamic Vent System (TVS), which simultaneously provides mixing and cooling in the liquid, and droplet spraying in the ullage region. Comprehensive models of the gas-droplet interaction have been developed for the thermal management of electronic equipment, evaporative mist flow heat exchangers ${ }^{12}$, and combustion engines ${ }^{13-16}$. However, applications of such models to cryogenic storage tanks have been quite scarce, and are limited to lumped models developed by NASA Marshall ${ }^{3}$ and Rockwell Aerospace ${ }^{17}$, as well as a Flow3D CFD model developed through a NASA-Boeing collaboration ${ }^{18}$. The latter predicted the experimental pressurization and pressure reduction trends well, but under predicted the experimental pressure rise by an average of $26 \%$, and under predicted the experimental pressure drop rates by an average of 50\%.

In previous papers, the results and validation of a comprehensive two-phase CFD model for simulating pressurization and pressure control experiments conducted in the Multipurpose Hydrogen Test Bed (MHTB) at the NASA Marshall Space Flight Center (MSFC) were presented by Kartuzova and Kassemi ${ }^{20,}{ }^{21}$. The $18-\mathrm{m}^{3}\left(693 \mathrm{ft}^{3}\right)$ storage tank employed in MHTB is representative of a fully integrated space transportation vehicle liquid hydrogen (LH2) propellant tank in both size and shape. The tank was designed to accommodate various components associated with different CFM concepts, including a spray-bar TVS system ${ }^{3}$ for active pressure control and a Multilayer Insulation (MLI) system for passive thermal isolation ${ }^{2}$.

In the two-phase CFD model of the MHTB test tank presented by Kartuzova and Kassemi ${ }^{21}$, the Volumeof-Fluid (VOF) technique was used to capture the shape and evolution of the interface. An Euler-Lagrange approach was utilized to track the spray droplets and capture the interaction between the droplets and the continuous phase (vapor). By coupling the droplet and VOF models, it was possible to perform particle tracking in the vapor, remove particles from the vapor domain when they reach the interface, and then add their contributions to the main liquid domain. An in-house droplet-ullage heat and mass transfer model ${ }^{20}$ was developed to account for changes in the tank pressure during the spraying cycle. In this model, the kinetic-based Schrage equation was used to calculate the evaporative/condensing mass transfer from/to the droplet. Unfortunately, as reported by the authors, the predicted tank pressure in this approach depends significantly on the accommodation coefficient used in the Schrage equation, the value of which is hard to determine ${ }^{21}$. Another limitation of utilizing the Schrage relation for calculating the droplet-ullage mass transfer is that, since the droplet temperature is calculated from an energy balance, it may deviate significantly from saturation conditions. While it is reasonable for a droplet to become slightly superheated as it travels through the warm vapor before it evaporates completely, a significant deviation from saturation conditions is intuitively questionable.

To overcome the limitations of the previously developed droplet-ullage mass transfer model, a new model was developed and is presented in the present paper. In this T-sat based model, a droplet is allowed to warm up to the saturation temperature corresponding to the vapor pressure, after which all of the heat provided by the vapor phase is consumed by the phase change process, while the droplet remains at its saturation temperature. Thus, the 
droplet evaporation rate is directly calculated from a droplet energy balance, and resorting to a kinetics mass transfer relationship, with its dependence on the value of the accommodation coefficient, is avoided. This approach also prevents significant deviations of the droplet temperatures from the saturation value. In what follows, the results of the present tank CFD model based on the T-sat droplet mass transfer approach are validated against the results of the MHTB spray cooling experiments for both the 50\% and 90\% fill ratios. The predictions of the present model are also compared with the results of the previous CFD model that used the Schrage equation for calculating the droplet mass transfer.

\section{Mathematical Model}

\section{A. Governing Equations}

The geometry and computational grid for the MHTB cryogenic storage tank partially filled with liquid hydrogen are shown in Fig. 1. This is a 3D $90^{\circ}$ sector grid, which was created from the $2 \mathrm{D}$ grid by revolving it along the tank's central axis. A 50\% tank fill ratio was considered. The fluid flow and heat transfer in the tank are described in terms of the continuity, Navier-Stokes, and energy equations for both phases:

$$
\begin{gathered}
\frac{\partial \rho}{\partial t}+\nabla(\rho \mathbf{v})=0 \\
\frac{\partial}{\partial t}(\rho \mathbf{v})+\nabla(\rho \mathbf{v v})=-\nabla p+\nabla\left[\mu_{e f f}\left(\nabla \mathbf{v}+\nabla v^{T}\right)\right]+\rho \mathbf{g}+\mathbf{F}_{v o l}, \\
\frac{\partial}{\partial t}(\rho E)+\nabla(\mathbf{v}(\rho E+p))=\nabla\left(k_{e f f} \nabla T\right)+S_{h} .
\end{gathered}
$$

In the present study, the liquid phase is treated as incompressible with variable temperature-dependent properties, except for the density. The liquid density is allowed to vary linearly with temperature in the body force term of the momentum equation according to the Boussinesq approximation. The vapor is modeled as a compressible ideal gas. All of the thermophysical and thermodynamic properties of the fluids are taken from the NIST Chemistry WebBook ${ }^{22}$ at saturation conditions.

In this study, the movement of the interface is captured diffusely by the model using the Volume of Fluid (VOF) method, as promulgated by Hirt and Nichols ${ }^{23}$. The interfacial energy, momentum, and mass balances are applied using source terms in the diffuse interfacial region.

\section{B. VOF Model}

In the VOF method, a volume fraction is defined in each cell such that the volume fractions of all of the phases sum to unity. In the cell, the change in the interface can be tracked by solving a continuity equation for the volume fraction of the $q^{\text {th }}$ phase:

$$
\frac{1}{\rho_{q}}\left[\frac{\partial}{\partial t}\left(\alpha_{q} \rho_{q}\right)+\nabla \cdot\left(\alpha_{q} \rho_{q} \mathbf{v}_{q}\right)=S_{\alpha_{q}}\right]
$$

where the volume fraction for the primary phase is determined from:

$$
\sum_{q=1}^{n} \alpha_{q}=1
$$

In the VOF method, the field variables and properties are defined in terms of the volume fraction, which for a general system can be written as:

$$
\rho=\sum_{q=1}^{n} \alpha_{q} \rho_{q}, \quad \mu_{e f f}=\sum_{q=1}^{n} \alpha_{q} \mu_{e f f_{q^{\prime}}} \quad k_{e f f}=\sum_{q=1}^{n} \alpha_{q} k_{e f f_{q}} .
$$

In this fashion, the continuity, momentum and energy equations, as described by Eq. (1) - (3), can be solved throughout the domain for the temperatures and velocities in the two phases. In the VOF model, the energy $(E)$ and temperature $(T)$ are treated as mass-averaged variables:

$$
E=\frac{\sum_{q=1}^{n} \alpha_{q} \rho_{q} E_{q}}{\sum_{q=1}^{n} \alpha_{q} \rho_{q}}
$$

where $E_{q}$ is based on the specific heat of the $q^{\text {th }}$ phase and the shared temperature.

Evaporation and condensation at the interface are modeled as a source term in the continuity equation for the volume fraction (Eq. 4), i.e.:

$$
S_{\alpha_{q}}=\dot{\mathbf{m}}_{i} \cdot \mathbf{A}_{i}
$$


where $\mathbf{A}_{i}$ is an interfacial area density vector, and $\dot{\mathbf{m}}_{i}$ is a mass flux vector, which for near equilibrium conditions can be determined based on the Schrage ${ }^{24}$ equation:

$$
|\dot{\mathbf{m}}|=\left(\frac{2 \sigma}{2-\sigma}\right)\left(\frac{M}{2 \pi R}\right)^{1 / 2}\left(\frac{P_{i}}{T_{i}^{1 / 2}}-\frac{P_{v}}{T_{v}^{1 / 2}}\right) .
$$

Here $\sigma$ is the accommodation coefficient; $M$ is the molar mass of the fluid; $R$ is the universal gas constant; $P_{i}$ and $P_{v}$ are, respectively, the interfacial and vapor pressures (it was assumed that $P_{i} \cong P_{s a t}$ ); $T_{i}$ and $T_{v}$ are, respectively, the interfacial and vapor temperatures (it was assumed that $T_{i}=T_{v} \cong T_{\text {sat }}$ at the interface). Finally, $\mathbf{A}_{\mathbf{i}}$ is defined as:

where $\alpha$ is the volume fraction of the primary phase.

$$
\mathbf{A}_{i}=|\nabla \alpha|
$$

In the present implementation, the surface tension forces at the interface are modeled via the Continuum Surface Force (CSF) model of Brackbill et al. ${ }^{25}$. In this model, the surface tension forces at the interface are transformed into a volume force $\left(\mathbf{F}_{v o l}\right)$, which is added as a source to the momentum equation:

$$
\mathbf{F}_{v o l}=\sum_{\text {pairs } i j, i<j} \sigma_{i j} \frac{\alpha_{i} \rho_{i} h_{i} \nabla \alpha_{j}+\alpha_{j} \rho_{j} h_{j} \nabla \alpha_{i}}{\frac{1}{2}\left(\rho_{i}+\rho_{j}\right)},
$$

where $h_{i}$ is the surface curvature calculated from the local gradients in the surface normal at the interface:

$$
h_{i}=\nabla \cdot \widehat{\mathbf{n}} .
$$

\section{Lagrangian Spray model}

A customized Lagrangian Spray model of the ANSYS Fluent CFD code ${ }^{26}$ was utilized for simulating the cooling of the MHTB tank, which was accomplished experimentally by spraying cold liquid into the vapor. This model uses the Euler-Lagrange approach, where the fluid phase (ullage) is treated as a continuum by solving the Navier-Stokes equations. The dispersed phase is solved by tracking a large number of particles (spray droplets) through the calculated flow field. The droplets exchange mass, momentum, and energy with the fluid phase. In the Lagrangian spray model, the droplet trajectory is calculated by integrating the force balance on the droplet. This force balance equates the droplet inertia with the forces acting on the droplet as:

$$
\frac{d \vec{u}_{p}}{d t}=F_{D}\left(\vec{u}-\vec{u}_{p}\right)+\frac{\vec{g}\left(\rho_{p}-\rho\right)}{\rho_{p}}+\vec{F}
$$

where $\vec{u}$ is the fluid phase velocity, $\vec{u}_{p}$ is the particle velocity, $\rho$ is the fluid density, $\rho_{p}$ is the density of the droplet, $F_{D}$ is the drag force per unit droplet mass, and $\vec{F}$ is an additional acceleration. The integration of time in equation 13 yields the velocity of the droplet at each point along the droplet trajectory. The spherical drag law proposed by Morsi and Alexander ${ }^{27}$ is applied for the droplet drag force calculation.

The droplet energy equation is solved in the user subroutine as:

$$
m_{p} c_{p_{p}} \frac{d T_{p}}{d t}=h \cdot A_{p}\left(T_{\infty}-T_{p}\right)-L \dot{m}_{p}
$$

where $m_{p}$ is the droplet mass, $c_{p_{p}}$ is the heat capacity of the droplet, $A_{p}$ is the surface area of the droplet, $T_{\infty}$ is the local temperature of the continuous phase, $h$ is the convective heat transfer coefficient, $L$ is the latent heat, and $\dot{m}_{p}$ is the rate of droplet evaporation or condensation in $\mathrm{kg} / \mathrm{s}$.

As mentioned before, two droplet mass transfer models are studied and compared in this paper. In the kinetic-based model, which was developed by the authors earlier ${ }^{20}$, the rate of droplet evaporation/condensation is obtained from the Schrage relation (eq. 9). The temperature of the droplet is then obtained from eq. 14. In the T-sat model, which is developed in this paper, the droplet is first allowed to warm up to the saturation temperature corresponding to the vapor pressure, after which it uses all of the energy supplied by the ullage for evaporation while it stays at the saturation temperature, such that:

$$
\begin{aligned}
& \text { if } T_{p}<T_{\text {sat }}\left(P_{v}\right)=>\dot{m}_{p}=0 ; T_{p} \text { is calculated from eq. } 14 \\
& \text { if } T_{p} \geq T_{\text {sat }}\left(P_{v}\right)=>T_{p}=T_{\text {sat }}\left(P_{v}\right) ; \dot{m}_{p} \text { is calculated from eq. } 14
\end{aligned}
$$

In this case, the evaporation rate can be calculated directly from the droplet energy balance (eq. 14). 
In both models, the correlation of Ranz and Marshall ${ }^{28,}{ }^{29}$ is used to evaluate the droplet heat transfer coefficient:

$$
N u=\frac{h d_{p}}{k_{\infty}}=2.0+0.6 \operatorname{Re}_{d}^{1 / 2} \operatorname{Pr}^{1 / 3},
$$

where $d_{p}$ is the droplet diameter, $k_{\infty}$ is the thermal conductivity of the continuous phase, $R e_{d}$ is the Reynolds number based on the particle diameter and the relative velocity, and $P r$ is the Prandtl number of the continuous phase.

The relative Reynolds number is calculated as:

$$
R e_{d} \equiv \frac{\rho d_{p}\left|\overrightarrow{u_{p}}-\vec{u}\right|}{\mu}
$$

where $\mu$ is the molecular viscosity of the fluid.

After the droplet energy equation is solved in the user subroutine, the mass, diameter, and temperature of each droplet are updated. The mass, momentum, and energy transfer between the droplets and the continuous phase is modeled via source terms added to equations 1-3.

The Lagrangian Spray model is coupled with the VOF model via a user subroutine which performs particle tracking in the ullage, removes particles from the ullage when they reach the interface, and then adds their contributions to the liquid mass, momentum, and energy equations through source terms. These source terms are added to those liquid cells near the location where the spray drops crossed the liquid-vapor interface. Because the VOF method produces a diffuse interface, the criteria for determining when the spray drops have crossed into the bulk liquid is defined as the liquid drop entering a fluid cell with a liquid volume fraction $>0.1$.

\section{Numerical Implementation}

In this study, the computational domain is discretized using an unstructured mesh of 184,440 cells, as depicted in Fig. 1. In both the ullage and liquid regions, the conservation equations are evolved in time using a bounded second order time stepping routine with a time step size on the order of $1 \times 10^{-2}$ seconds with the implicit VOF model. The second order monotone upwinding scheme is used to discretize the convective fluxes in the momentum, energy, and turbulence equations. The PISO method is used for the pressure-velocity coupling.

For the volume fraction equation, the Compressive discretization scheme is used. The Compressive scheme is a second order reconstruction scheme for the VOF equation based on the slope limiter ${ }^{26}$. In this scheme:

$$
\phi_{f}=\phi_{d}+\beta \nabla \alpha_{d}
$$

where $\phi_{f}$ is the face value of the volume fraction, $\phi_{d}$ is the donor cell value of the volume fraction, $\beta$ is the slope limiter, and $\nabla \alpha_{d}$ is the donor cell volume fraction gradient.

During the spray cooling of the tank, cold liquid enters the liquid phase as a "tiny jet," which is modeled as a source term defined in a user subroutine. This source term is added to the mass, energy and momentum equations in Fluent. The results for the spray cooling of the tank with the $90 \%$ liquid fill ratio were presented by the authors earlier $^{20}$. The new droplet mass transfer model, where the droplet temperature is set to the saturation temperature at the tank pressure (T-sat), was applied in the $90 \%$ fill ratio case. However, the main focus of this study is on the $50 \%$ fill ratio cases. In the $50 \%$ fill ratio case, there are 22 liquid jets in the liquid region, and 21 spray injections in the vapor region. Both the liquid jets and the spray injections have the same uniform temperature of $21.088 \mathrm{~K}$, and a variable liquid flow rate ranging from 0 to $7.5447 \mathrm{e}-4 \mathrm{~kg} / \mathrm{s}$. The spray injection type is a plain orifice atomizer with four particle streams per injection. Two-way coupling between the droplets and the continuous phase is enabled. In order to model the heat and mass transfer between the droplets and the ullage, a value for the mass transfer rate is first calculated in the subroutine. Then the droplet energy equation is solved, and the droplet temperature is updated. The mass and diameter of the droplet are updated to account for the amount of the evaporated or condensed mass. At this point, the corresponding mass and energy sources are added to the ullage. set to $1 \times 10^{-8}$.

The convergence criteria are set to $1 \times 10^{-5}$ for all of the equations except the energy equation, for which it is

\section{Results and Discussion}

The simulation case studies presented in this paper are based on the MHTB tank self-pressurization and spray bar mixing experiments (Test Segment P263981T) conducted in normal gravity ${ }^{3}$. The MHTB tank consists of a cylindrical mid-section with a $3.05 \mathrm{~m}$ diameter and $3.05 \mathrm{~m}$ height and two 2:1 elliptical end caps. A schematic of the tank with the spray-bar holes and pump locations is shown in Fig. 1b. There are 22 holes in the liquid region, which are modeled as liquid jet point sources for mass, momentum, and energy, and 21 holes in the vapor region, 
which are modeled as spray injections using the plain orifice atomizer model, with four particle streams per injection. Area averaged sink terms are added for mass, momentum and energy at the pump location to account for liquid recirculating in the TVS system. Liquid hydrogen is injected into the vapor with constant properties at $\mathrm{T}=$ $21.088 \mathrm{~K}$. The spray-bar hole diameter is $1.7 \mathrm{e}-03 \mathrm{~m}$ with a flow-through length of $0.711 \mathrm{e}-03 \mathrm{~m}$. The total liquid flow rate into the spray-bar assembly is based on measured flow rate data ${ }^{3}$ and varies with time. In the simulations, the liquid flow rate is distributed uniformly to all spray-bar holes ( 43 holes in each of the four spray-bar tubes).

The gravitational vector is aligned with the tank's central axis in the negative axial direction. A uniform heat flux of $0.89873 \mathrm{~W} / \mathrm{m}^{2}$ was applied at the inside of the tank wall in the vapor region, and $2.0841 \mathrm{~W} / \mathrm{m}^{2}$ in the liquid region. These values are based on a previous thermal analysis and on multi-node simulations. Conduction through the tank wall was not considered. The spray bar assembly is approximated as lying along the tank's central axis. Its wall is treated as an adiabatic surface.

The 3D $90^{\circ}$ sector grid used for the spray-bar mixing simulations is shown in Fig. 1a. It is created from the 2D axisymmetric grid by revolving it along the tank's central axis. It has 20 nodes in the azimuthal direction. The grid is refined near the interface, tank wall, and spray-bar wall. A grid independence study was performed for the $50 \%$ fill ratio self-pressurization case; the results were presented earlier ${ }^{20}$.

The experiment was conducted with three different tank liquid fill ratios $(25 \%, 50 \%$ and $90 \%)$.The CFD results for the $50 \%$ and $90 \%$ tank fill ratio were presented by the authors earlier ${ }^{20}$. 21 . Here, a detailed comparison between the previous kinetic based model for calculating the droplet mass transfer using the Schrage equation, and the newly developed T-sat based model that assumes that the droplet remains at the saturation temperature and calculates the mass transfer from the droplet via a droplet energy balance, is conducted for the $50 \%$ fill ratio case. Only the first spray on/off cycle after self-pressurization in Test Segment P263981T is simulated, during which no liquid is directed into the Joule Thomson device, and thus the temperature of the liquid exiting the spray bar is essentially the same as the temperature of the liquid entering the pump located near the tank bottom.

In the cases with the 50\% tank fill ratio, the experimental temperature field at the beginning of mixing was used as an initial condition for the simulations. In the cases with the $90 \%$ tank fill ratio, the predicted temperature and velocity fields at the end of a prior axisymmetric tank self-pressurization simulation were interpolated into a 3D $90^{\circ}$ sector grid, and were used as the initial conditions for simulating the spray-bar mixing cooling cycle. The initial tank pressure matched the experimental one for each case.

Detailed comparisons between the results of the kinetic and T-sat based droplet mass transfer models for the 50\% fill ratio case are presented in Fig. 2 - 11. Figures $2-7$ display the predicted temperature distributions of the continuous phases at the center plane of the injections, together with the evolving droplet temperatures at different time sequences during the injection/mixing cycle. Figures $8-11$ show comparisons among the temperature evolutions at different locations in the vapor and liquid as predicted by the two models and as measured in the experiment.

The predicted vapor phase temperature contours and droplet temperatures on the center plane of the injection, at $\mathrm{t}=1 \mathrm{sec}$ after the initiation of spraying, are shown respectively in Figs. 2 and 3 for the kinetic-based (Schrage) and T-sat based models. Note that the vapor and droplets are each colored according to their respective temperature scales, which are common between the two figures, to allow a direct comparison between the two figures. Results for the kinetic-based droplet model shown in Fig. 2 indicate that the droplets emerging from the spray bar are initially colder than the vapor, but as they travel through the ullage and towards the interface, their temperatures increase noticeably due to heating by the vapor. Note that the droplets that are injected at the top of the tank travel through a region of warmer vapor due to the thermal stratification in the tank that was established during the preceding tank self-pressurization period. Therefore, they are at a higher temperature than the droplets injected from the lower sections of the spray-bar. As shown in Fig. 2, the droplet temperatures increase significantly from $21.13 \mathrm{~K}$ to $33.88 \mathrm{~K}$ in this case. The vapor temperature contours and droplet temperatures at the center plane of injections after 1 second of spraying, as calculated by the T-sat droplet model, are shown in Fig. 3. The results indicate the same general droplet behavior as in the previous case, but a comparison of Figs. 2 and 3 shows that the droplet temperatures calculated by the T-sat model are significantly lower than those calculated by the kinetic model; they only vary between 21.14 and $22.05 \mathrm{~K}$. As expected, in the T-sat model, after the droplet temperature approaches its saturation value, all of the heat transfer from the vapor to the droplet is consumed by the phase change process, and as a result, the droplets will be close to their saturation temperatures during most of their travel through the ullage.

The temperature contours at the center plane of the spray bar holes calculated by the kinetic-based Schrage and $\mathrm{T}$-sat models at three different times during the spray-bar mixing $(\mathrm{t}=1,15$, and 34 seconds) are shown in Figures 4 and 5 respectively. The results indicate that the spray effectively mixes the vapor and lowers the vapor temperature after 34 seconds of spraying. Even though both models predict similar temperature distributions in most 
of the vapor region, the T-sat model predicts lower vapor temperatures in the areas with the highest droplet concentrations.

Figures 6 and 7 display droplets colored by temperature along their trajectory after 1,15 and 34 seconds of spraying, as predicted by the kinetic-based and T-sat models, respectively. The Schrage model predicts cold droplets entering the tank at the injection location. The droplets warm up as they travel through the vapor, especially at the center of the vapor region, as shown in Fig. 6. The region covered by warm droplets is largest after 15 seconds of spraying, as compared to the beginning of spraying at 1 second. At 34 seconds of spraying, the droplets are also significantly cooler than at 15 seconds, because the vapor region has already cooled down by the ensuing heat transport and mixing process. The droplet temperatures predicted by the kinetic model vary between 21.13 and $33.88 \mathrm{~K}$. The droplet temperatures predicted by the T-sat model vary between 21.14 and $22.05 \mathrm{~K}$. The range and values of the droplet temperatures predicted by the T-sat model are much less than the ones predicted by the kinetic model, as discussed before.

The temperature was measured at various locations in the vapor and liquid regions in the experiment. The temperature evolutions at three different measurement locations in the vapor region and one location in the liquid region, calculated by the kinetic and T-sat droplet mass transfer models, are compared against each other and against the experimental data in Fig. 8-11. The temperature evolutions at the measurement location at the top of the vapor region (TD1) are plotted in Fig. 8. At this location, both models capture the slope of the temperature decline in the experiment with similar fidelity. More oscillations in the vapor temperatures are evident in the CFD simulations, as predicted by both models, than are measured in the experiment. At this location (TD1), the T-sat model predicts temperatures that are slightly lower than the ones predicted by the Schrage model.

Fig. 9 shows a comparison between the temperature evolutions in the middle of the vapor region (at the temperature measurement location TD4) predicted by the Schrage and T-sat models and from experimental data. Both models predict a similar slope for the temperature decrease in the vapor, which agrees well with the experimental one. The T-sat model, however, still predicts lower temperatures compared to the kinetic model. At this location, more temperature oscillations are again predicted by the CFD simulations than are measured during the experiment.

A comparison of the temperature evolutions at the bottom of the vapor region near the interface (at temperature measurement location TD6) predicted by the two models and measured during the experiment is shown in Fig. 10. At this location, both models agree very well with the experiment for the first 30 seconds of mixing. After 30 seconds of mixing, the kinetic-based model agrees well with the experiment, but the T-sat model under predicts the vapor temperatures slightly. At this location, oscillations in the vapor temperatures measured in the experiment are higher than the ones predicted by the CFD models between $t=10$ and 25 seconds after the initiation of mixing.

Finally, the temperature evolutions in the liquid (TD7) are compared between the Schrage and T-sat models and the experiment in Fig.11. The liquid temperature remains constant in both the experiment and the CFD predictions. Here, the temperatures predicted by the CFD models agree very well with experimental data.

It is interesting to examine the impact of the different droplet heat and mass transfer evolutions as predicted by the two models on the overall tank pressure drop. The evolution of the tank pressure simulated by using the kinetic-based Schrage and T-sat models for the 50\% fill ratio are compared against experimental data in Fig. 12. The T-sat model shows excellent agreement with the corresponding experimental results, while the Schrage model over predicts the tank pressures considerably. This trend is also confirmed for the $90 \%$ fill ratio case as displayed in Fig. 13, where tank pressure evolutions calculated by the two droplet models are again compared against experimental data.

During spray injection, the ullage pressure is affected by two main phenomena: heat and mass exchange with the droplet. The T-sat droplet mass transfer model limits the extent of the temperature rise in the droplet, as seen in Figs. 5-6, by assuming that the temperature of the droplets will remain at the saturation value, and that most of the heat removed from the vapor is consumed during the evaporation process in the form of latent heat. However, the kinetic-based droplet mass transfer model limits the extent of the evaporative mass transfer from the droplet according to the value dictated by the Schrage equation, and assumes that the remainder of the heat transferred from the ullage to the droplet is used to raise the internal energy, and hence the temperature, of the droplet. Thus the temperature difference between the droplet and the ullage, and consequently the heat loss from the ullage to the droplet predicted by the T-sat model, are much larger than those predicted by the kinetic-based model. Conversely, the T-sat based model also predicts a larger mass transfer to the ullage than the kinetic-based model. Since the T-sat based model agrees well with the measured rate of the pressure drop in the tank during the spraying cycle, while the kinetic-based Schrage model over predicts the measured values considerably, as shown in Fig. 12 and 13, this seems to be a testimony to the fact that the effects of heat transfer overwhelm those of mass transfer during tank pressure control. 
Another shortcoming of the kinetic-based Schrage model is that it is significantly affected by the value of the accommodation coefficient used for calculating the droplet mass transfer. Since available data on the values of the accommodation coefficient is scarce, especially for cryogenic fluids, the new T-sat model, which does not use an accommodation coefficient, has an inherent and distinct advantage.

\section{Conclusion}

A CFD model for the cooling of cryogenic storage tanks by spraying cold liquid into the vapor was developed $^{20}$ and applied for modeling the MHTB spray bar mixing experiment. An Eulerian-Lagrangian approach implemented in the spray model of the ANSYS Fluent CFD code was utilized to track the spray droplets, and to capture the interaction between the droplets and the continuous phase (ullage). The spray model was coupled with the VOF model via a user subroutine which performs particle tracking in the vapor, removes particles from the vapor domain when they reach the interface, and then adds their contributions to the liquid through source terms.

A new T-sat based model for calculating the droplet heat and mass transfer was developed and validated against spray cooling in the MHTB tank experiments with 50\% and 90\% fill ratios. The results of this model are presented and compared with the results of the previously developed kinetic-based droplet mass transfer model. In the new model, a droplet is allowed to warm up to the saturation temperature corresponding to the tank vapor pressure, and then evaporate while staying at the saturation temperature. In the previously developed model, the kinetic-based Schrage equation was used for calculating the droplet mass transfer, and the droplet temperature was calculated from a droplet energy balance. This allowed the droplet to warm up to a temperature considerably above saturation. The predictions of the kinetic-based model were significantly affected by the hard-to-determine value of the accommodation coefficient used in the Schrage equation. The T-sat model does not have this limitation, since the droplet mass transfer is computed indirectly from the droplet energy balance.

The tank pressure evolutions during the spray period predicted by the two models were compared with the experimental data for the 50\% and $90 \%$ tank fill ratios. The results of the new T-sat model agreed better with the experimental data for the pressure evolution in the tank for both fill ratios, as compared to those of the previously presented kinetic-based Schrage model, which over predicts the tank pressures significantly. A detailed comparison of the tank temperature and flow fields, as well as the droplet temperatures and distribution, predicted by the two models was presented. Both models showed that the droplets reduce the temperature and promote mixing in the vapor region via heat and mass exchange during the spray cycle. The kinetic-based model showed that the temperatures of the droplets increase as they travel through the vapor towards the interface. In the case with the Tsat model, the temperature of the droplets did not vary significantly. Therefore, the T-sat model predicted more cooling of the vapor as compared to the kinetics model. This resulted in a higher pressure decay rate in the tank, and a closer agreement with the experimental results.

\section{Acknowledgments}

This work was supported by the NASA Space Technology Mission Directorate's Technology Demonstration Missions Program under the Evolvable Cryogenics Project.

\section{References}

${ }^{1}$ Salzman, J., "Fluid management in space-based systems," Proceedings of the Engineering, Construction, and Operations in Space, 5th international conference on space, Vol. 1, 1996, p. 521-6.

${ }^{2}$ Martin, J.; and Hastings, L.: "Large-Scale Liquid Hydrogen Testing of a Variable Density Multilayer Insulation with a Foam Substrate,” NASA/TM-2001-211089, Marshall Space Flight Center, AL, June 2001.

${ }^{3}$ Hastings, L.J., Flachbart, R.H., Martin, J.J., Hedayat, A., Fazah, M., Lak, T., Nguyen, H., Bailey J.W. "Spray Bar ZeroGravity Vent System for On-Orbit Liquid Hydrogen Storage” NASA TM-212926, 2003.

${ }^{4}$ Barsi, S. and Kassemi, M. "Investigation of Tank Pressurization and Pressure Control-Part II: Numerical Modelling", ASME Journal of Thermal Science \& Engineering Applications, Vol. 5, No 2, pp- 041006: 1-9, December 2013

${ }^{5}$ Kartuzova, O., Kassemi M., "Modeling Interfacial Turbulent Heat Transfer during Ventless Pressurization of a Large Scale Cryogenic Storage Tank in Microgravity," AIAA 47 th Joint Propulsion Conference, AIAA, San Diego, CA, 2011

${ }^{6}$ Grayson, G.D., Lopez, A., Chandler, F.O., Hastings L.J., Tucker, S.P., "Cryogenic Tank Modeling for the Saturn AS-203 Experiment," Proceedings of the 42 ${ }^{\text {nd }}$ AIAA/ASME/SAE/ASEE Joint Propulsion Conference, AIAA 2006-5258, 2006.

${ }^{7}$ Barsi, S., Kassemi, M., "Numerical and Experimental Comparisons of the Self-Pressurization Behavior of an LH2 Tank in Normal Gravity", Cryogenics, 48(3/4), pp. 122-129, 2007.

${ }^{8}$ Kartuzova, O., Kassemi, M., "Modeling Active Pressure Control in a Large Scale Cryogenic Storage Tank in Normal Gravity," AIAA 48 $8^{\text {th }}$ Joint Propulsion Conference, AIAA, Atlanta, GA, 2012 
${ }^{9}$ Panzarella, C.H. and Kassemi, M., Comparison of Several Zero-Boil-Off Pressure Control Strategies for Cryogenic Storage in Microgravity, Journal of Power \& Propulsion, Vol. 25, No. 2, pp. 424-434, 2009.

${ }^{9}$ Panzarella, C.H. and Kassemi, M., Pressure Control of Large Cryogenic Tanks in Microgravity, Cryogenics, Vol. 44/6-8, pp. 475-483, 2004

${ }^{9}$ Panzarella, C.H. and Kassemi, M., On the Validity of Purely Thermodynamic Description of Two-Phase Cryogenic Storage Tank, Journal of Fluid Mechanics, Vol 484, pp.136-148, 2003.

${ }^{12}$ Kumari, N. Bahadur, V., Hode, M., Salamon, T., Kolodner, P, Lyons, A., Garimella, S. V., “Analysis of evaporating mist flow for enhanced convective heat transfer", International Journal of Heat and Mass Transfer 53 (2010) 3346-3356.

${ }^{13}$ Sirignano, W.A., "Fluid Dynamics of Sprays," Journal of Fluids Engineering, Vol. 115, no. 3, pp. 345-378, September 1993.

${ }^{14}$ Crowe, C.T., Sharma, M.P., and Stock, D.E., "The Particle-Source-in Cell (PSI-CELL) Model for Gas-Droplet Flows", $J$. Fluids Eng., Vol. 99, pp. 325, 1977.

${ }^{15}$ Raju, M.S., and Sirignano, W.A., "Spray Computations in a Centerbody Combustor" Journal of Engineering for Gas Turbines and Power, Vol. 1, No. 4, pp. 710-718, October 1989.

${ }^{16}$ Raju, M.S., "Current Status of the Use of Parallel Computing in Turbulent Reacting Flows, Computations Involving Sprays, Scalar Monte Carlo Probability Density Function \& Unstructured Grids", Advances in Numerical Heat Transfer, Vol. 2, ch. 8, pp.259-287, 2000.

${ }^{17}$ Nguyen, H.: Zero-G “Thermodynamic Venting System (TVS) Performance Prediction Program”, Rockwell Aerospace, Contract NAS8-39202, May 24, 1994

${ }^{18}$ Grayson et al, "CFD Modeling of Helium Pressurant Effects on Cryogenic Tank Pressure Rise Rates in Normal Gravity", AIAA 2007-5524, 43rd AIAA/ASME/SAE/ASEE Joint Propulsion Conference \& Exhibit - 11 July 2007, Cincinnati, OH,

${ }^{19}$ Menter, F. R., "Two-Equation Eddy-Viscosity Turbulence Models for Engineering Applications," AIAA Journal, Vol. 32 No. 8, 1994, pp. 1598-1605

${ }^{20}$ Kartuzova, O., Kassemi, M., Agui, J., Moder, J. "Self-Pressurization and Spray Cooling Simulations of the Multipurpose Hydrogen Test Bed (MHTB) Ground-Based Experiment," AIAA 50 th Joint Propulsion Conference, AIAA, Cleveland, OH, 2014

${ }^{21}$ Kartuzova, O., Kassemi, M. "CFD Modeling of the Multipurpose Hydrogen Test Bed (MHTB) Self-Pressurization and Spray Bar Mixing Experiments in Normal Gravity: Effect of the Accommodation Coefficient on the Tank Pressure," AIAA 51 ${ }^{\text {st }}$ Joint Propulsion Conference, AIAA, Orlando, FL, 2015

${ }^{22}$ Lemmon, E.W., McLinden, M.O., and Friend, D.G., "Thermophysical Properties of Fluid Systems" in NIST Chemistry WebBook, NIST Standard Reference Database Number 69, Eds. Linstrom, P.J., and Mallard, W.G., National Institute of Standards and Technology, Gaithersburg MD, 20899, http://webbook.nist.gov.

${ }^{23}$ Hirt, C.W., and Nichols B.D., "Volume of fluid (VOF) method for the dynamics of free boundaries," Journal of Computational Physics, Vol. 39 No. 1, 1981, pp. 201-225.

${ }^{24}$ Schrage, R.W., A theoretical study of interphase mass transfer, Columbia University Press, New York, 1953.

${ }^{25}$ Brackbill J.U., Kothe, D.B., Zemach, C., "A continuum method for modeling surface tension,” J. Comp. Phys. Vol. 100, 1992, pp. 335-354.

${ }^{26}$ ANSYS Fluent Documentation. Release 16.0. November 2014.

${ }^{27}$ S. Morsi, A. and Alexander, A. J. "An Investigation of Particle Trajectories in Two-Phase Flow Systems," Journal of Fluid Mechanics, Vol. 55, No. 2, 1972, pp. 193-208.

${ }^{28}$ Ranz, W. E. and Marshall, W. R., Jr. "Evaporation from Drops, Part I," Chemical Engineering Progress, Vol. 48, No. 3, 1952, pp. 141-146.

${ }^{29}$ Ranz, W. E. and Marshall, W. R., Jr. "Evaporation from Drops, Part II," Chemical Engineering Progress, Vol. 48, No. 4, 1952, pp. 173-180. 


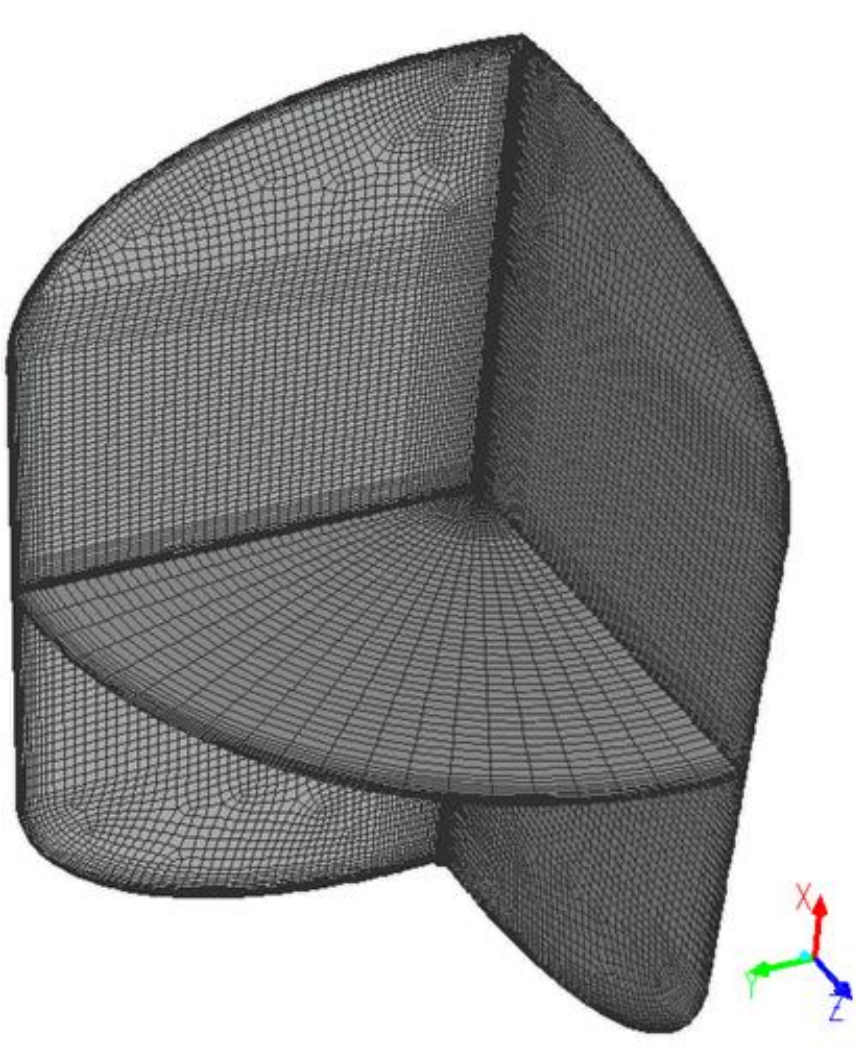

(a)

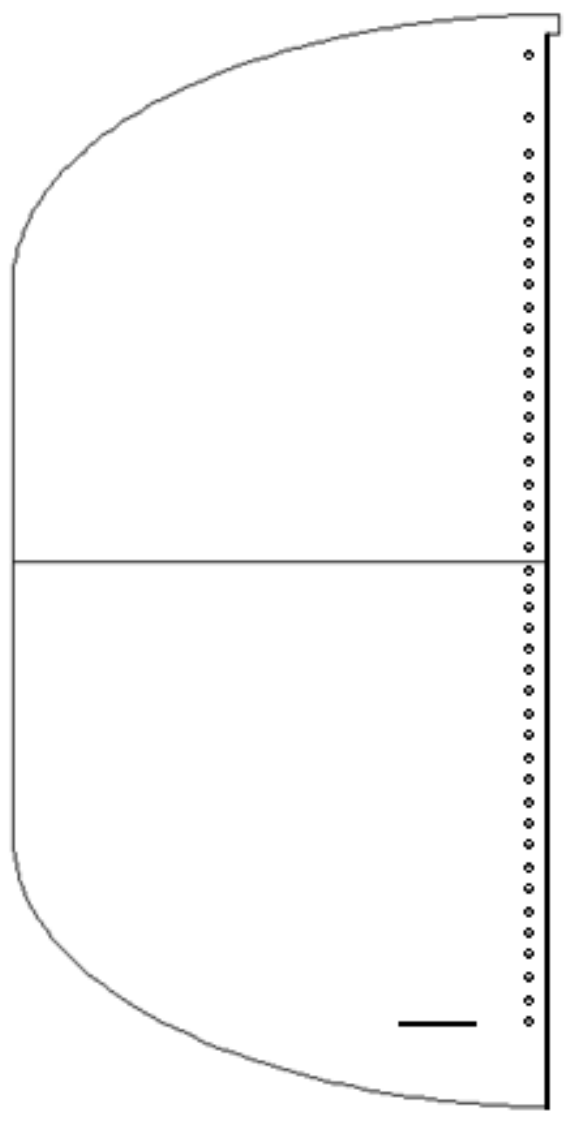

(b)

Figure 1: 3D $90^{\circ}$ sector grid used for the spray bar mixing cases (184,440 cells) (a); and a schematic of spray bar holes and pump locations at the center plane of the injections (b) 


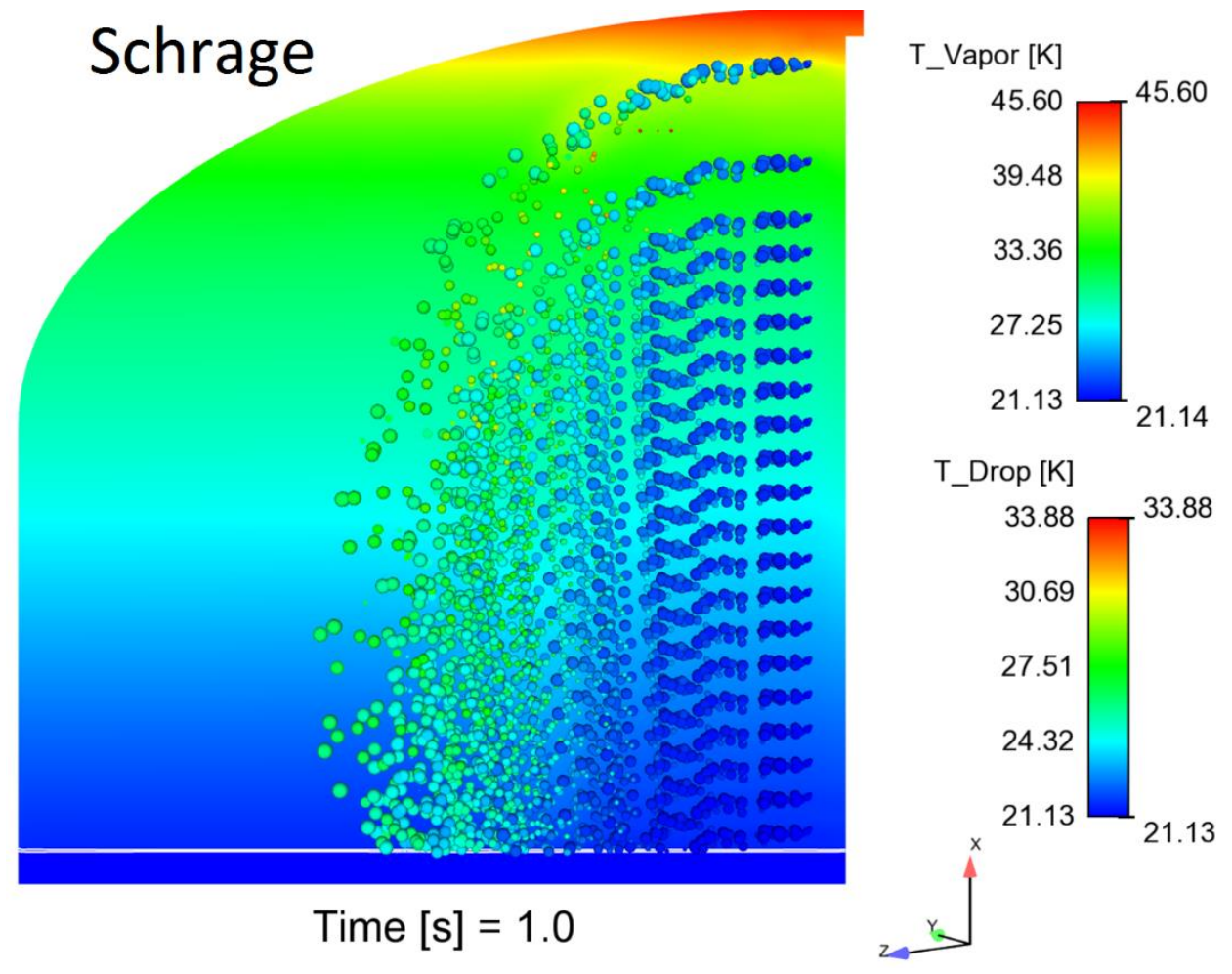

Figure 2: Temperature of the vapor at the center plane of the injections and droplets colored by the droplet temperatures after 1 second of spray (Schrage droplet mass transfer model)

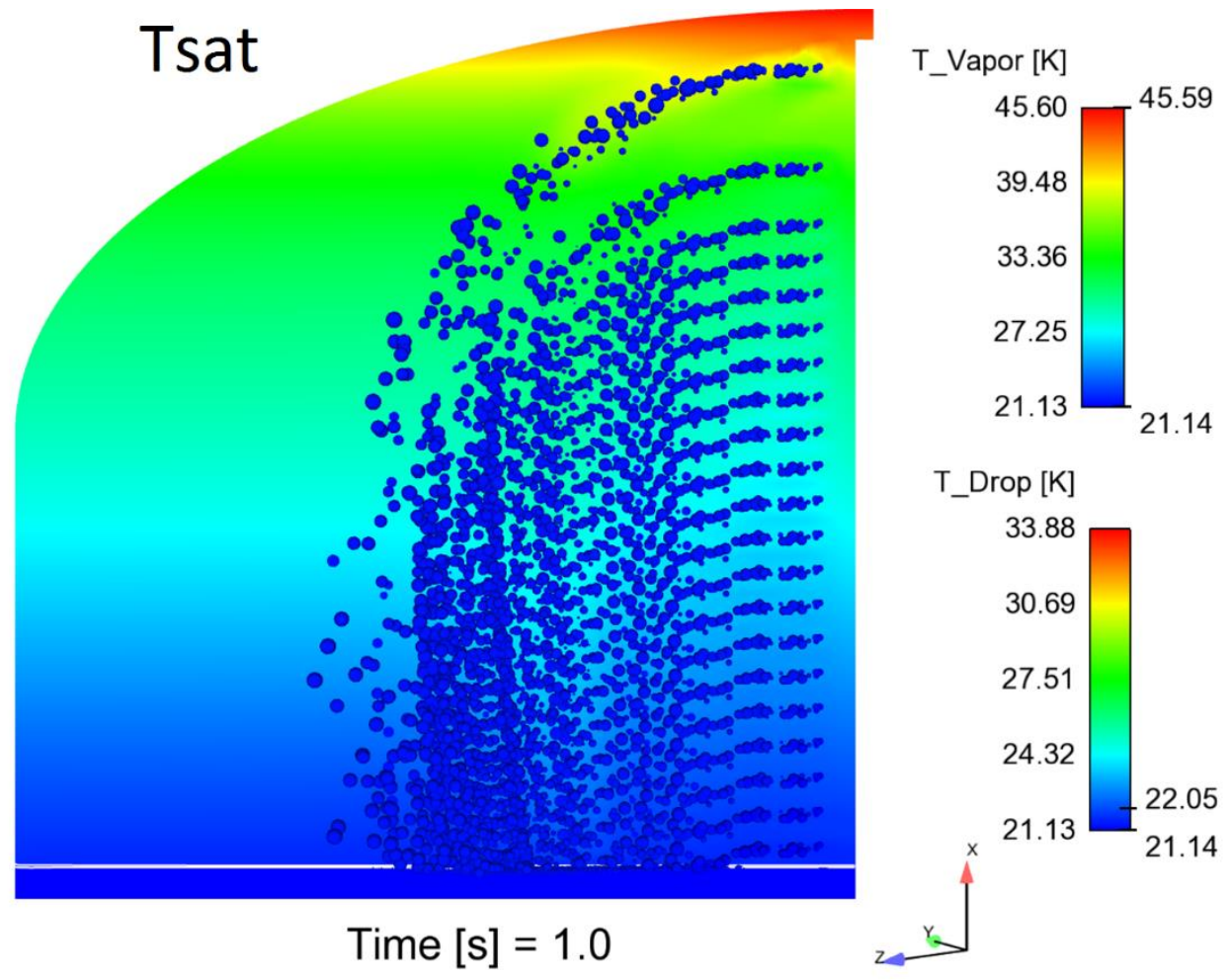

Figure 3: Temperature of the vapor at the center plane of the injections and droplets colored by the droplet temperatures after 1 second of spray (T-sat droplet mass transfer model) 


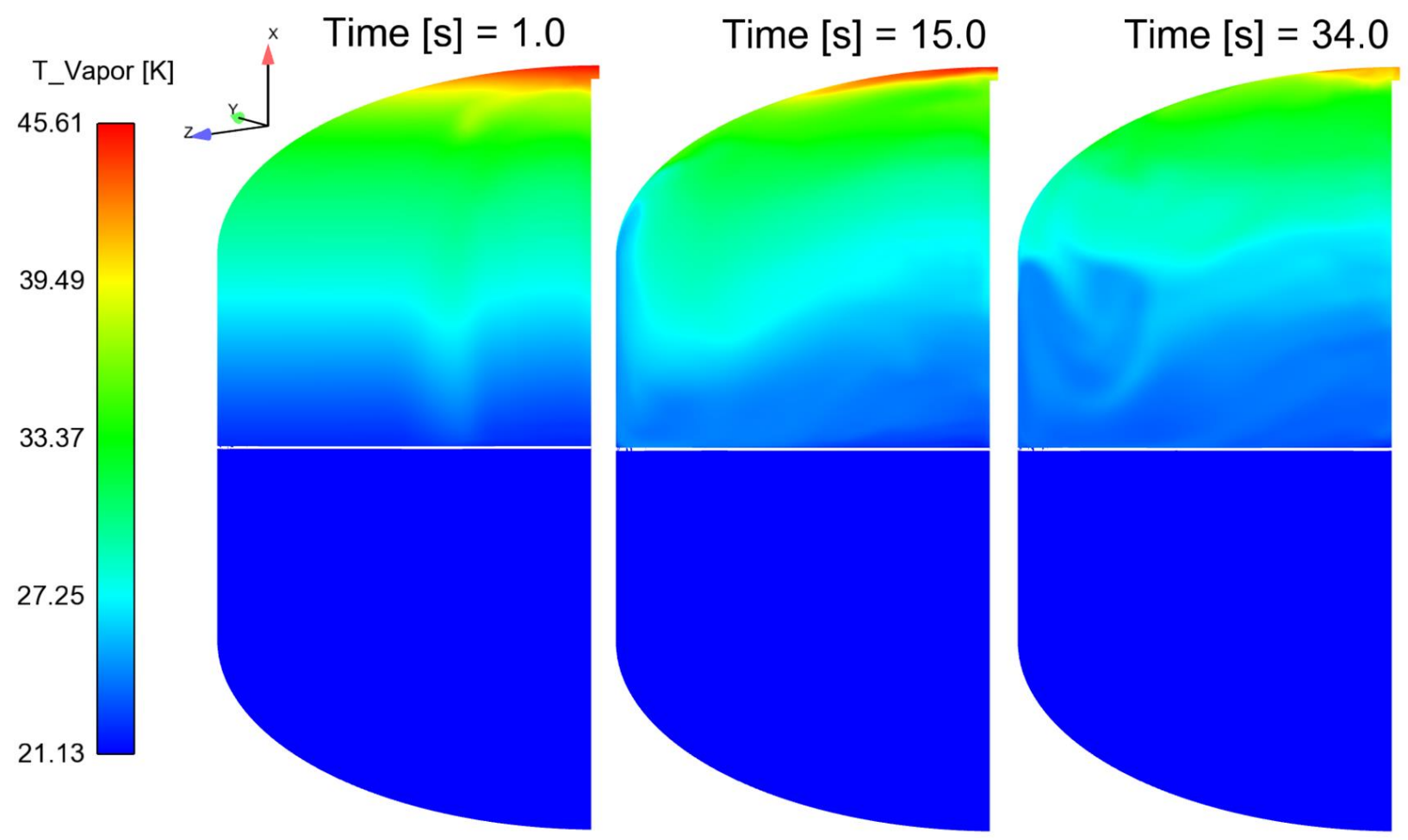

Figure 4: Temperature in the vapor at the center plane of injections at 1; 15 and 34 seconds of spray (Schrage droplet mass transfer model)

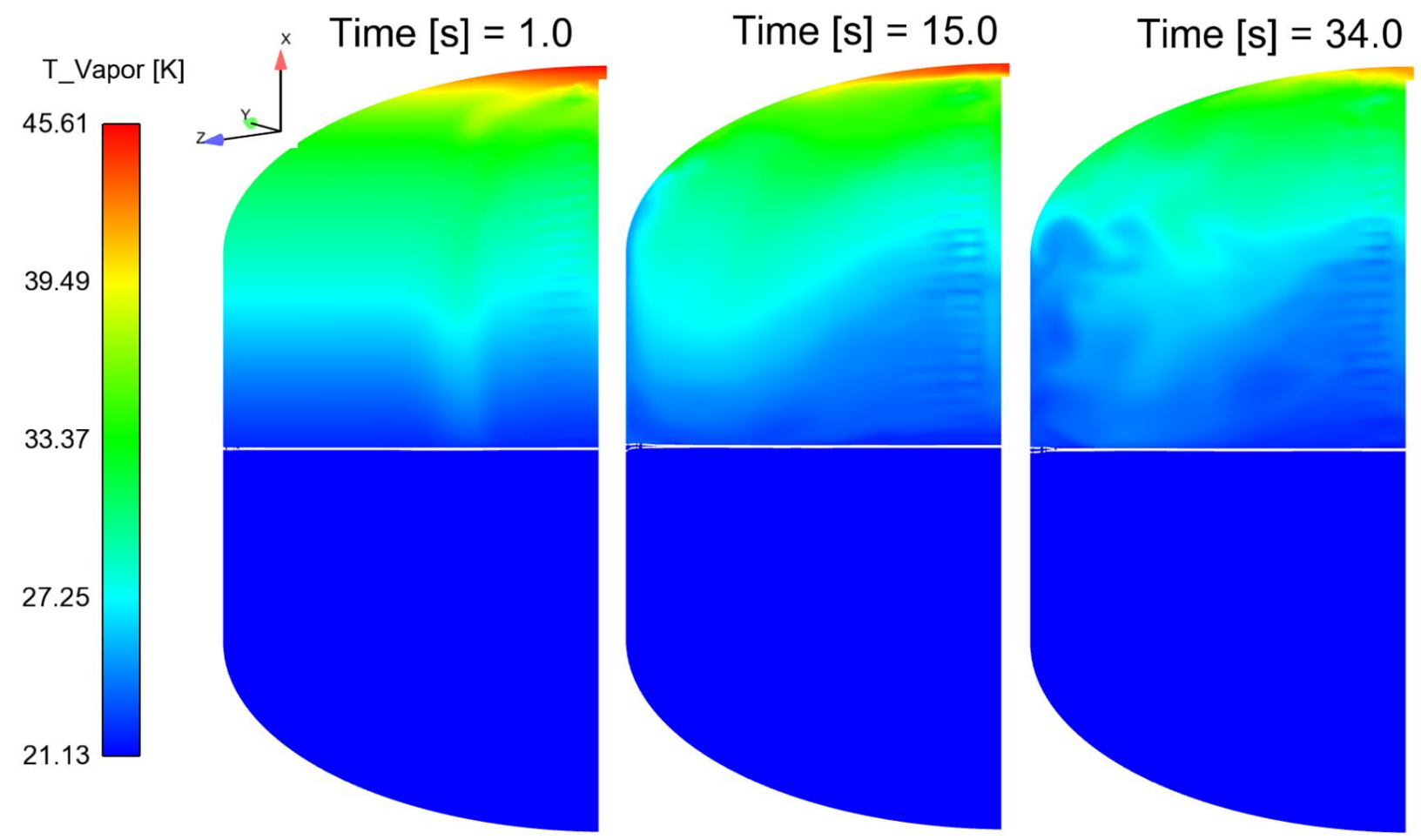

Figure 5: Temperature in the vapor at the center plane of injections at 1;15 and 34 seconds of spray (T-sat droplet mass transfer model) 

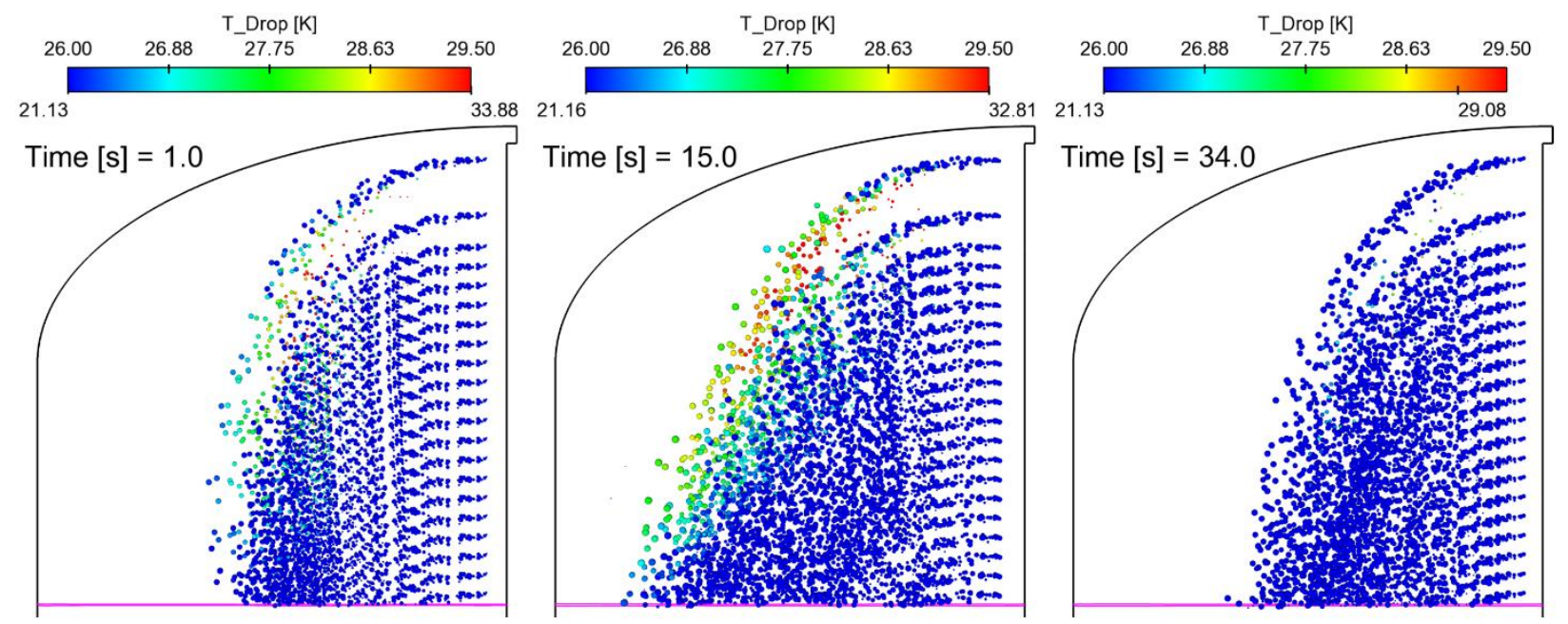

Figure 6: Droplets colored by temperatures at the center plane of injections at 1; 15 and 34 seconds of spray (Schrage droplet mass transfer model)

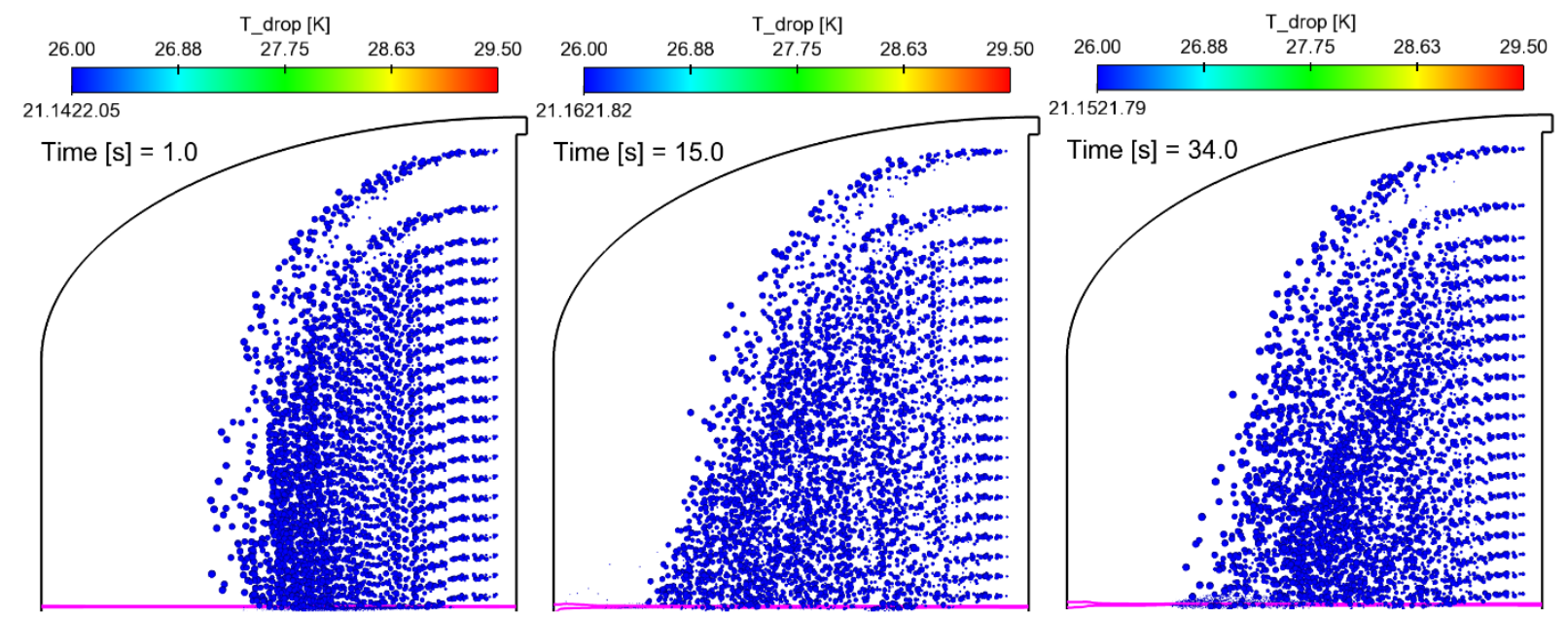

Figure 7: Droplets colored by temperatures at the center plane of injections at 1; 15 and 34 seconds of spray (T-sat droplet mass transfer model) 


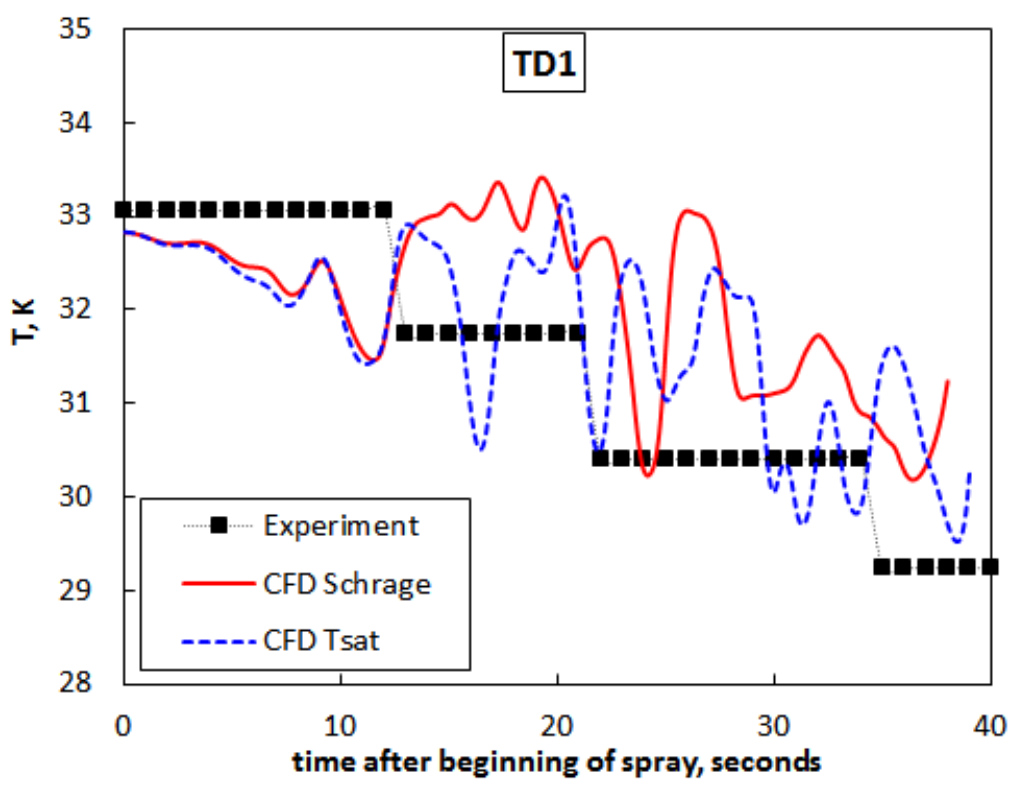

(a)

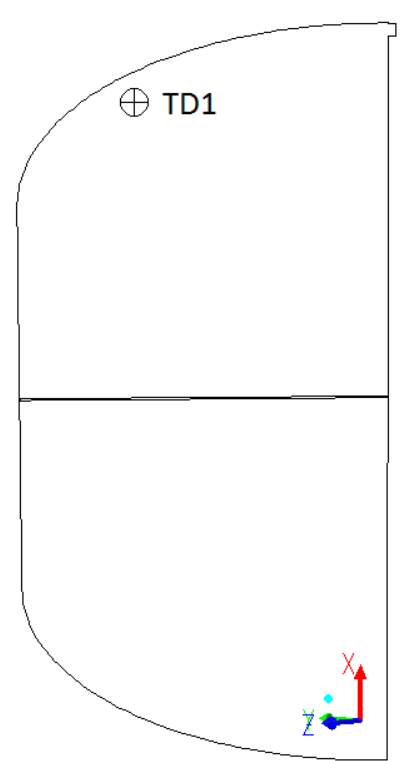

(b)

Figure 8: Temperature evolutions for TD1 compared between the T-sat and Schrage droplet mass transfer models and experiment (a); Schematic of the tank with TD1 location shown (b)

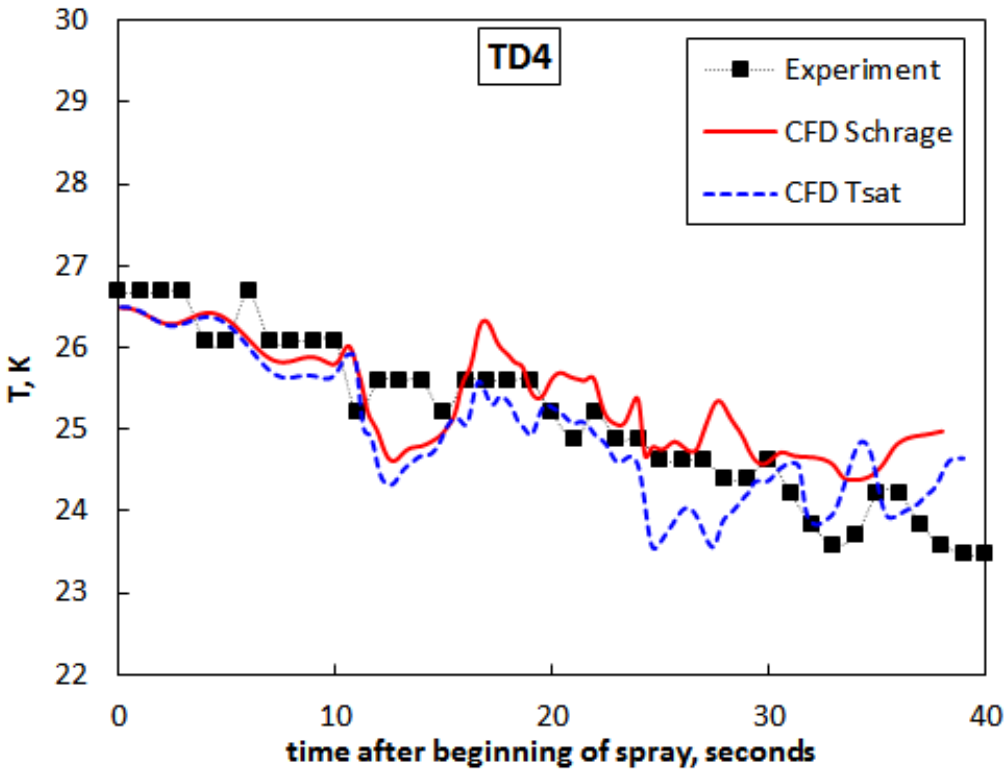

(a)

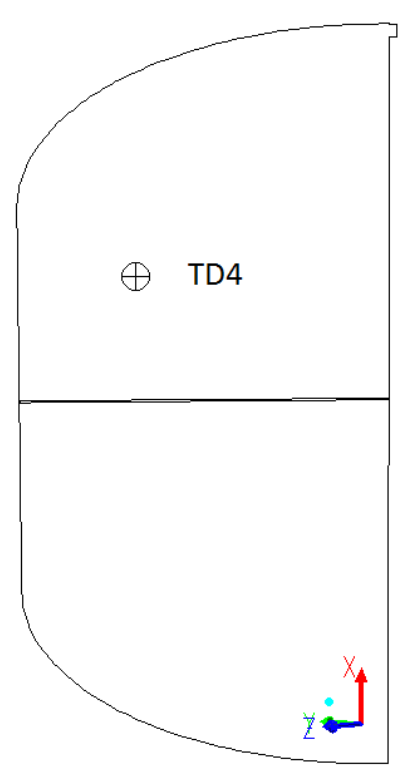

(b)

Figure 9: Temperature evolutions for TD4 compared between the T-sat and Schrage droplet mass transfer models and experiment (a); Schematic of the tank with TD4 location shown (b) 


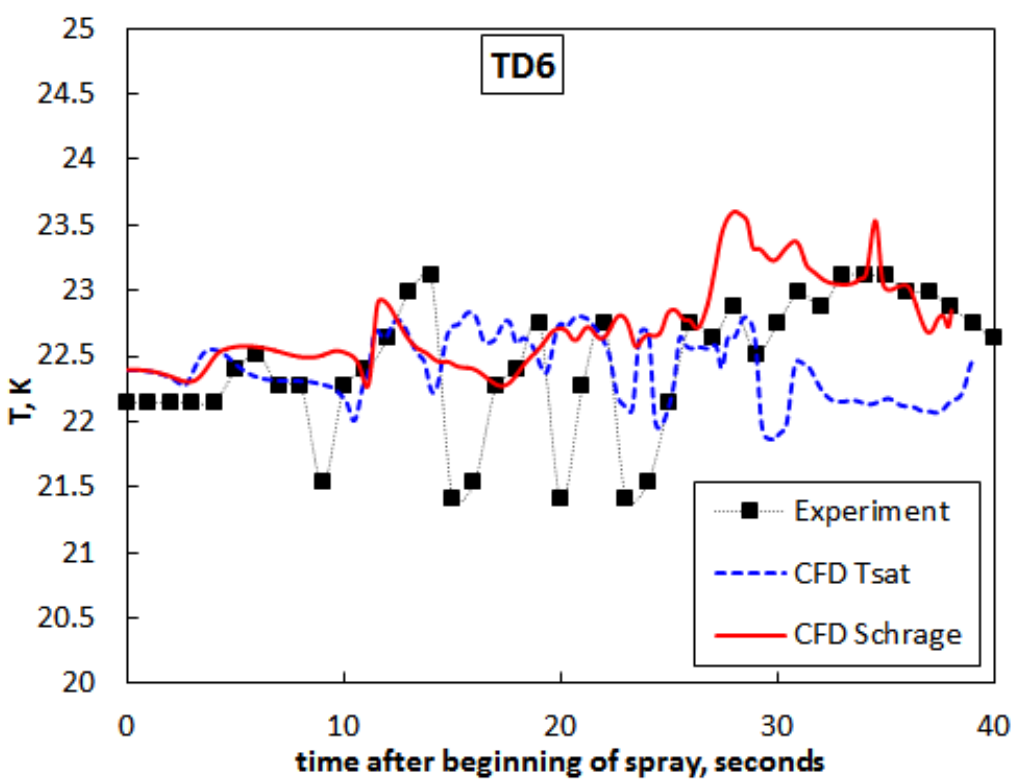

(a)

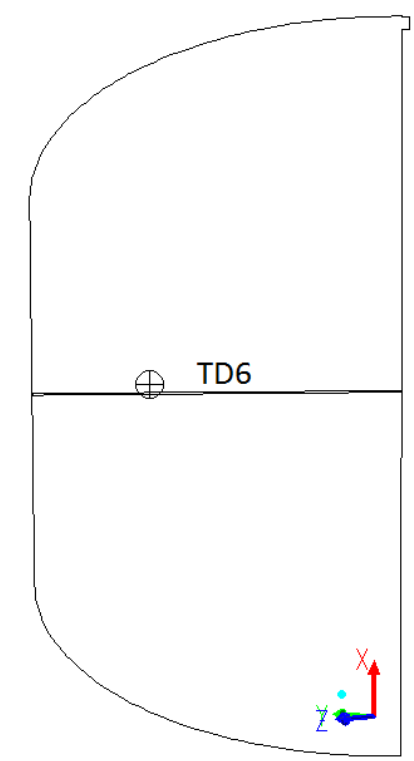

(b)

Figure 10: Temperature evolutions for TD6 compared between the T-sat and Schrage droplet mass transfer models and experiment (a); Schematic of the tank with TD6 location shown (b)

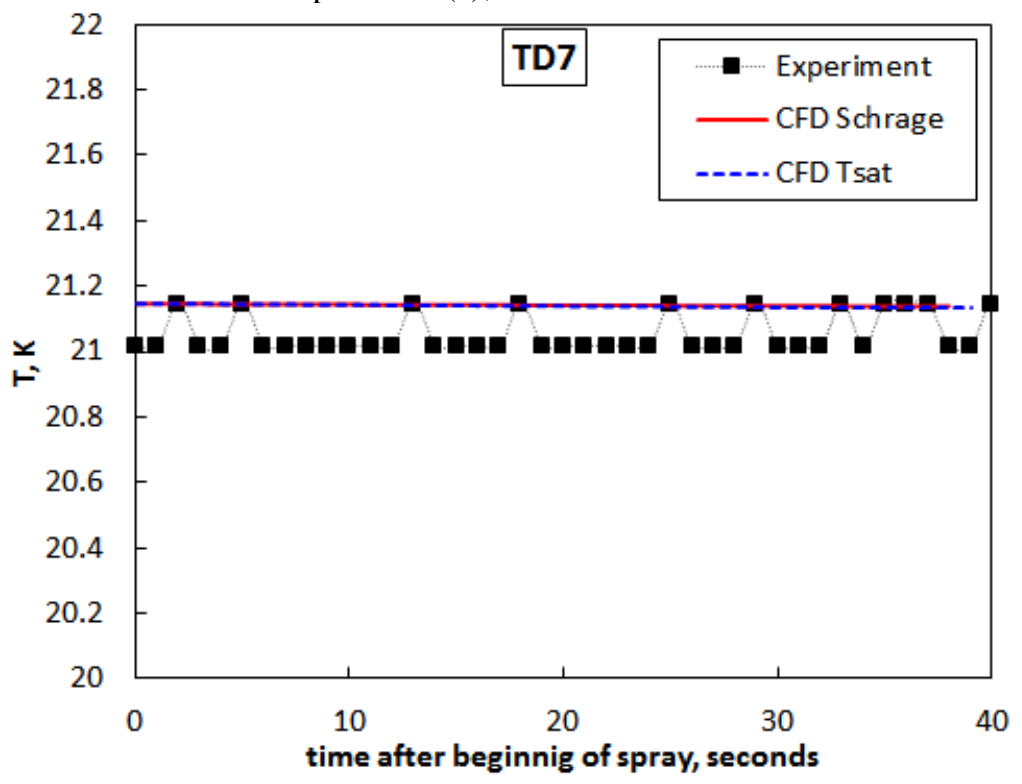

(a)

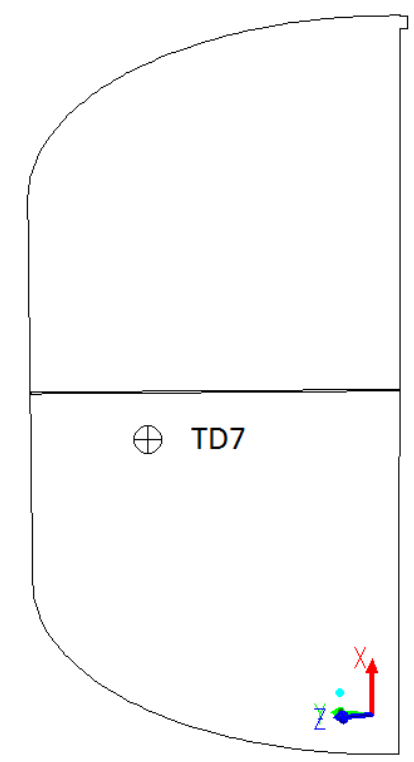

(b)

Figure 11: Temperature evolutions for TD7 compared between the T-sat and Schrage droplet mass transfer models and experiment (a); Schematic of the tank with TD7 location shown (b) 


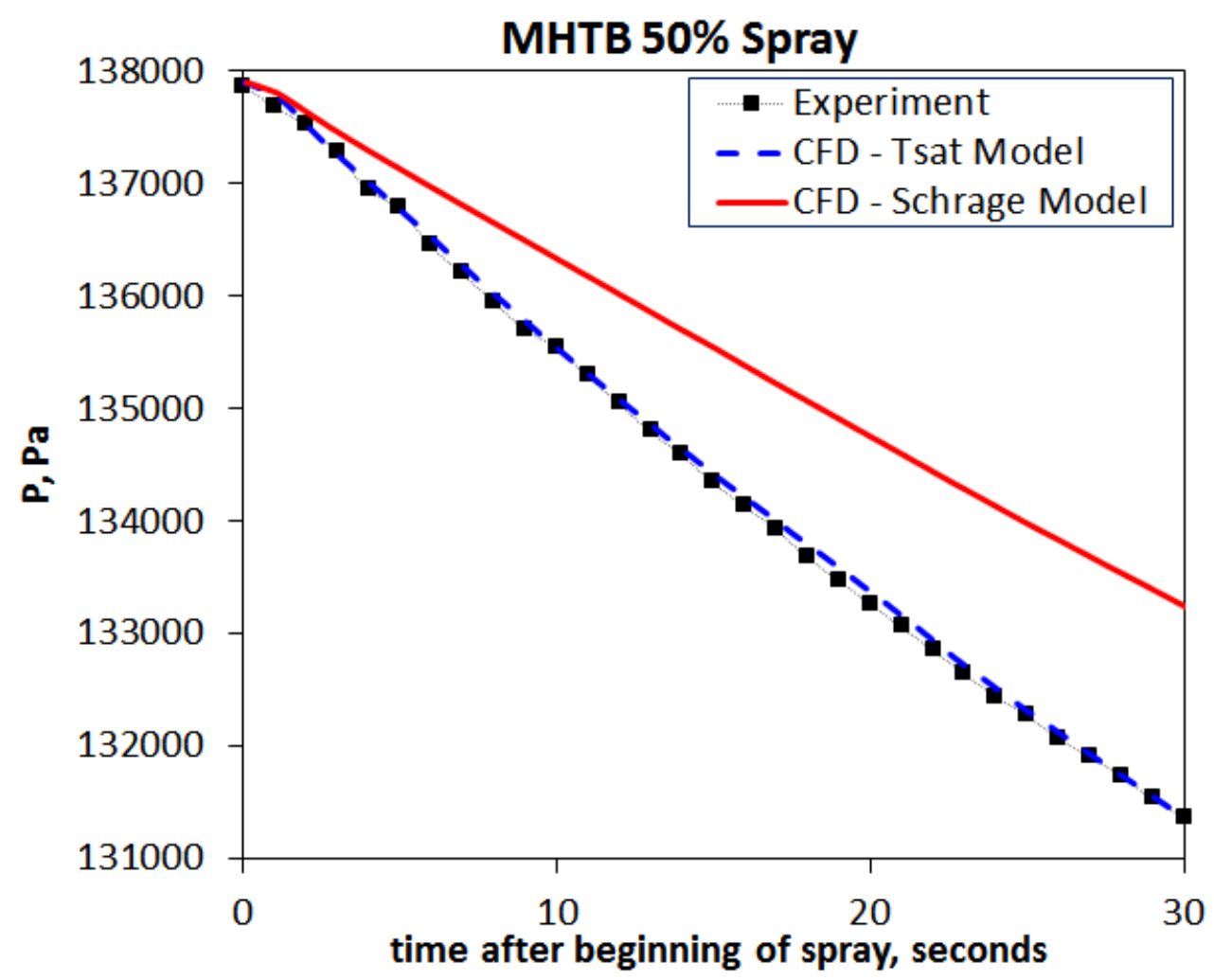

Figure 12: Tank pressure evolution during spray cooling for the $50 \%$ fill ratio case

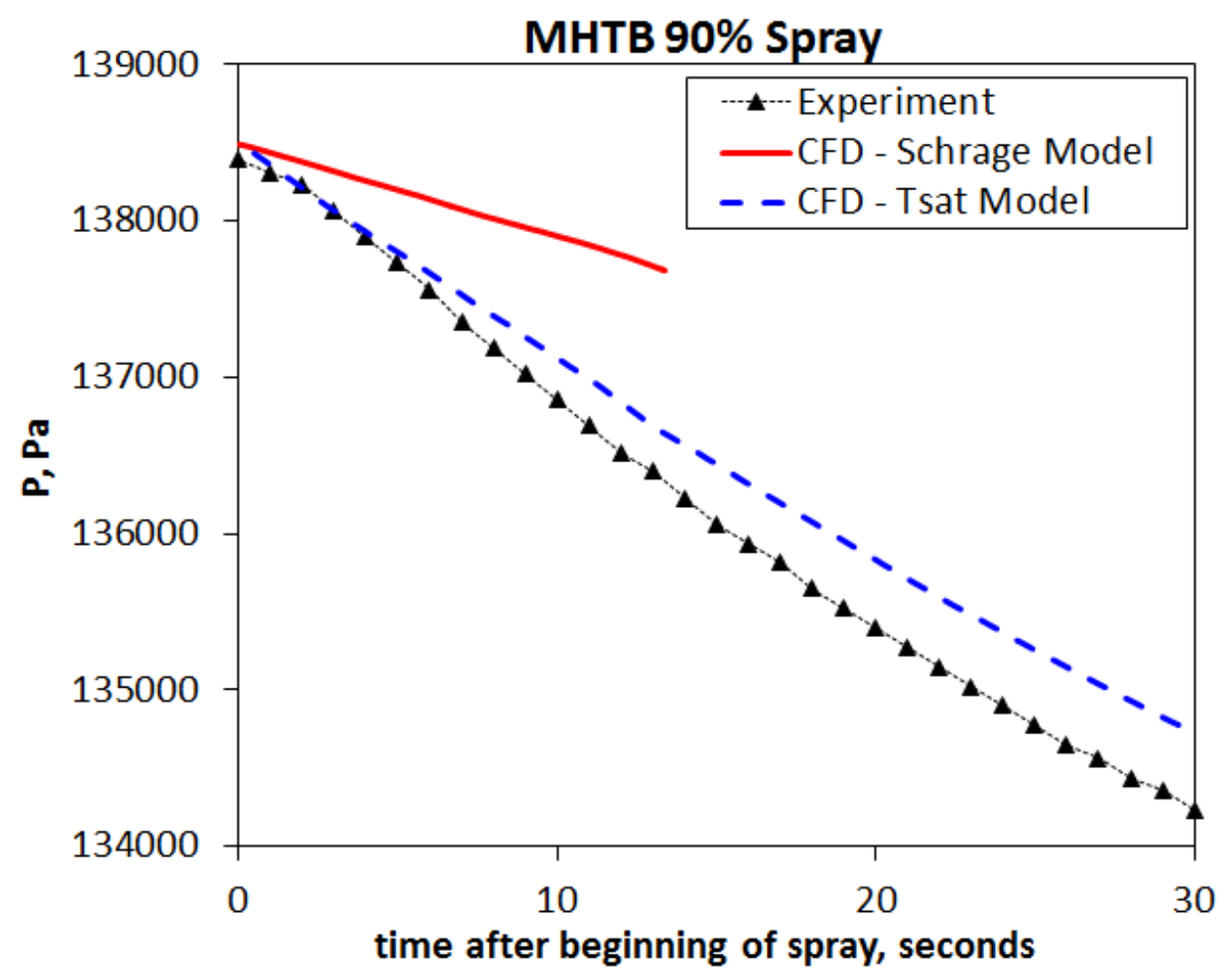

Figure 13: Tank pressure evolution during spray cooling for the $90 \%$ fill ratio case 16

American Institute of Aeronautics and Astronautics 